\title{
BMJ Open Evaluation of volatile sedation in the postoperative intensive care of patients recovering from heart valve surgery: protocol for a randomised, controlled, monocentre trial
}

\author{
Armin Niklas Flinspach (D) , ${ }^{1}$ Eva Herrmann, ${ }^{2}$ Florian Jürgen Raimann (D) ,' \\ Kai Zacharowski, ${ }^{1}$ Elisabeth Hannah Adam (i) ${ }^{1}$
}

To cite: Flinspach AN, Herrmann E, Raimann FJ, et al. Evaluation of volatile sedation in the postoperative intensive care of patients recovering from heart valve surgery: protocol for a randomised, controlled monocentre trial. BMJ Open 2022;12:e057804. doi:10.1136/ bmjopen-2021-057804

- Prepublication history and additional supplemental material for this paper are available online. To view these files, please visit the journal online (http://dx.doi.org/10.1136/ bmjopen-2021-057804).

Received 27 September 2021 Accepted 21 January 2022

Check for updates

(c) Author(s) (or their employer(s)) 2022. Re-use permitted under CC BY-NC. No commercial re-use. See rights and permissions. Published by BMJ.

${ }^{1}$ Department of Anaesthesiology, Intensive Care Medicine and Pain Therapy, University Hospital Frankfurt, Goethe University Frankfurt, Germany, Klinikum der Johann Wolfgang GoetheUniversität Frankfurt, Frankfurt am Main, Hessen, Germany ${ }^{2}$ Department of Biostatistic and Mathematic Modeling, Goethe University, Frankfurt, Germany, Goethe-Universitat Frankfurt am Main, Frankfurt am Main, Hessen, Germany

Correspondence to Dr Armin Niklas Flinspach; armin.flinspach@kgu.de

\section{ABSTRACT}

Introduction Patients undergoing heart valve surgery are predominantly transferred postoperatively to the intensive care unit (ICU) under continuous sedation. Volatile anaesthetics are an increasingly used treatment alternative to intravenous substances in the ICU. As subject to inhalational uptake and elimination, the resulting pharmacological benefits have been repeatedly demonstrated. Therefore, volatile anaesthetics appear suitable to meet the growing demands of fast-track cardiac surgery. However, their use requires special preparation at the bedside and trained medical and nursing staff, which might limit the pharmacological benefits. The aim of our work is to assess whether the temporal advantages of recovery under volatile sedation outweigh the higher effort of special preparation. Methods and analysis The study is designed to evaluate the differences between intravenous sedatives $(n=48)$ and volatile sedatives $(n=48)$ in continued intensive care sedation. This study will be conducted as a prospective, randomised, controlled, single-blinded, monocentre trial at a German university hospital in consenting adult patients undergoing heart valve surgery at a university hospital. This observational study will examine the necessary preparation time, staff consultation and overall feasibility of the chosen sedation method. For this purpose, the continuation of sedation in the ICU with volatile sedatives is considered as one study arm and with intravenous sedatives as the comparison group. Due to rapid elimination and quick awakening after the termination of sedation, closer consultation between the attending physician and the ICU nursing staff is required, in addition to a prolonged setup time. Study analysis will include the required setup time, time from admission to extubation as primary outcome and neurocognitive assessability. In addition, possible operation-specific (blood loss, complications), treatment parameters (catecholamine dosages, lung function) and laboratory results (acute kidney injury, acid base balance (lactataemia), liver failure) as influencing factors will be collected. The studyrelevant data will be extracted from the continuous digital records of the patient data management system after the patient has been discharged from the ICU. For statistical
Strengths and limitations of this study

- Prospective randomised controlled trail of the realtime clinical benefit of the use of volatile sedation on nurse and physician workloads for time to patient awakening.

- Extend limited data on the short-term use of volatile sedatives in intensive care units in the setting of planned postoperative sedation.

- Application of volatile sedatives after cardiac valve surgery requiring heart-lung machine use with all authorised commercial anaesthetic conserving devices.

- Limited follow-up period covering up to hospital discharge.

- Limited by monocentric implementation at a university centre of cardiosurgical care and the associated intensive care unit.

evaluation, 95\% Cls will be calculated for the median time to extubation and neurocognitive assessability, and the association will be assessed with a Cox regression model. In addition, secondary binary outcome measures will be evaluated using Fisher's exact tests. Further descriptive and exploratory statistical analyses are also planned. Ethics and dissemination The study was approved by the Institutional Ethics Board of the University of Frankfurt, Germany (\#20-1050). Informed consent of all individual patients will be obtained before randomisation. Results will be disseminated via publication in peer-reviewed journals.

Trial registration number Clinical trials registration (NCT04958668) was completed on 1 July 2021.

\section{INTRODUCTION}

Cardiac valve surgery remains a complex operative procedure under cardiopulmonary bypass (CPB), which is associated with a significant risk of postoperative complications such as bleeding, renal failure, arrhythmias or neurocognitive impairments. ${ }^{1}$ 
Therefore, patients undergoing valve reconstruction or valve replacement are usually postoperatively transferred to the intensive care unit (ICU) under continuous sedation. ${ }^{2}$ To maintain the required (analogue) sedation during ICU therapy, various substances are commonly used, such as volatile anaesthetics or intravenous sedatives combined with opioids. ${ }^{34}$

Volatile anaesthetics have been indispensable for maintaining adequate sedation in the perioperative phase for many decades and can also be used successfully under CPB. Their ruinous usage in ICUs is limited due to technical reasons. ${ }^{5-8}$

Intravenous sedatives, such as the frequently used substances propofol, clonidine and midazolam, undergo complex pharmacological metabolisation and elimination mechanisms. In contrast, volatile anaesthetics are subject to inhalational uptake and elimination and, for example, in the case of isoflurane, are nearly eliminated without metabolisation $(0.2 \%){ }^{9}$ The use of both intravenous and inhaled anaesthetics has been proven to be a safe therapeutic standard for decades. The pharmacological advantages, in terms of the low context-sensitive halflife of volatile anaesthetics, have been repeatedly shown to be beneficial with respect to the recovery of postoperative patients. Accordingly, the use of volatile sedatives in ICUs has been included in sedation recommendations since $2015 .^{1011}$

Technical advances over the last two decades have led to the development of technical devices that enable the use of volatile anaesthetics in ICUs. The development of reflector membranes was crucial. ${ }^{12}$ These enabled counter financially viable use through anaesthetic gas recovery of up to $90 \%$. Currently, two commercial systems are offered for this purpose: the AnaConDa system (Sedana Medical $\mathrm{AB}$, Danderyd, Sweden) and the MIRUS system (TIM Medical, Koblenz, Germany). ${ }^{81314}$

The use of volatile sedatives in ICUs requires specific bedside preparation as well as trained medical and nursing staff. This includes, in particular, the preparation of the anaesthetic conserving device with an anaesthetic gas collection container, which results in increased time required for setting up the intensive care bed.

As surgical treatments have evolved, it has become easier to accomplish the same therapeutic goals with management options that do not require extended postoperative mechanical ventilation. Accordingly, the opportunity to reduce the morbidity associated with prolonged ventilation and shorten the length of stay in the ICU is being driven forward with the development of 'fast-track' cardiac surgery. The aim of the fast-track procedure is to extubate patients recovering from open heart surgery within the first 6 postoperative hours. ${ }^{41516}$ To enable immediate neurological assessment and extubation after stabilisation of the patient's condition, a considerable interval for redistribution processes (especially with regard to the context-sensitive half-life time) and elimination of the substances must be taken into account, depending on the duration of the preceding intravenous sedation.

The postoperative use of volatile anaesthetics in intensive care has already shown a significant acceleration of extubation for patients undergoing bypass surgery. ${ }^{3}$

\section{Aims}

While the use of volatile anaesthetics in ICUs is becoming an increasingly common clinical practice, there is a lack of prospective randomised trials on the extent to which known pharmacological advantages can be realised, despite the higher nursing and medical effort required. Our intended study would investigate the use of volatile anaesthetics as an established sedation option with regard to the wake-up behaviour of patients undergoing heart valve surgery. It will be investigated to what extent the already described positive effects can be realised, despite the necessity of differentiated intensive care and medical management. This primarily includes the necessity of workplace conversion to the previously described anaesthetic-conserving devices.

\section{METHODS AND ANALYSIS \\ Study design}

The study has been designed to assess the effect of continuing intensive care sedation with intravenous compared with volatile sedatives in patients after they receive heart valve surgical procedures. The study will be conducted as a prospective, randomised controlled, single-blinded, monocentric trial at a university hospital. The start of study recruitment is planned for 1 September 2021. The study is expected to enrol 48 patients per study arm over a period of up to 2 years. Block randomisation will be stratified according to single or combined surgical interventions and based on randomisation lists generated at the Institute of Biostatistics and Mathematical Modelling of Goethe University Frankfurt. The randomisation lists will be blinded to the study investigators before treatment arm allocation, and the block size will remain confidential during the study. Patients will be treated in a single-blinded approach (see figure 1).

\section{Patient population}

Patients presenting to the Department of Anaesthesiology, Intensive Care Medicine and Pain Therapy for preoperative evaluation prior to heart valve surgery in the Department of Cardiac and Vascular Surgery will be screened with regard to their suitability based on the following defined inclusion and exclusion criteria.

\section{Inclusion criteria}

- Adult (>18 years old) patients.

- Admission to the ICU after cardiac valve surgery.

- Informed consent of participant.

Exclusion criteria

- Age $<18$ years. 


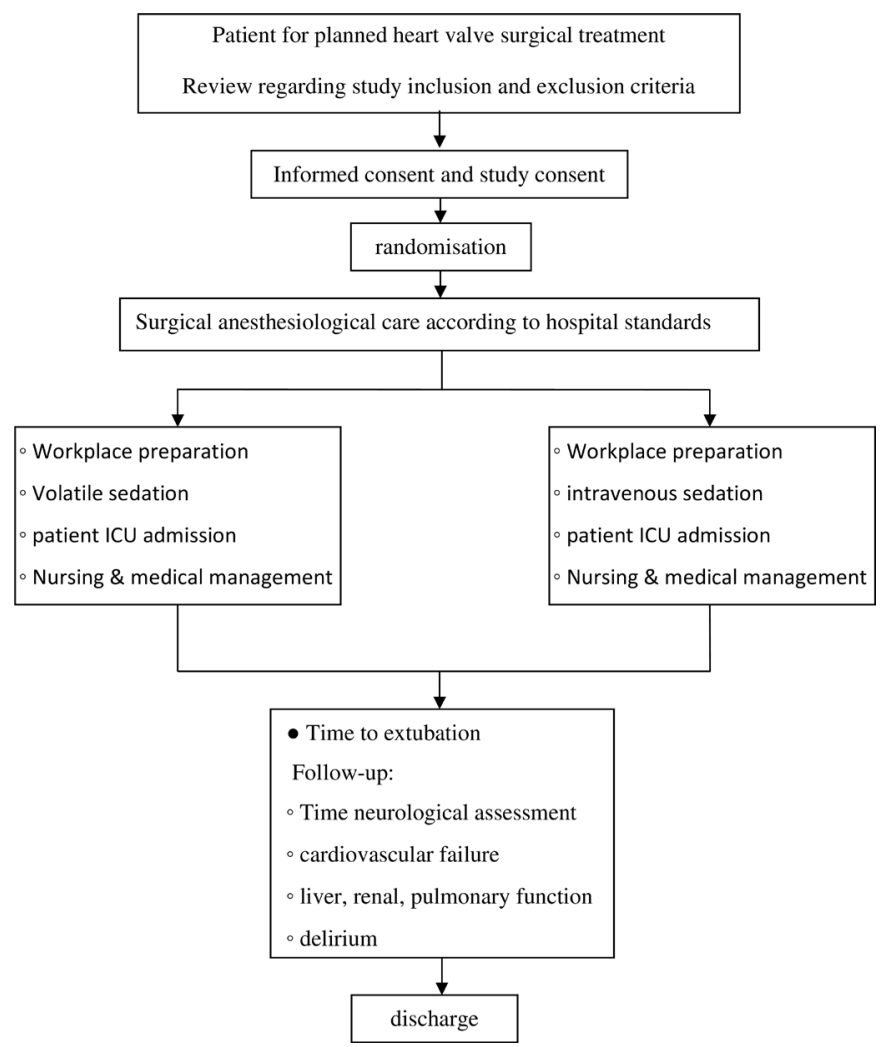

Figure 1 Flow chart of the study design for the evaluation of volatile sedation in postoperative intensive care in patients recovering from heart valve surgery. ICU, intensive care unit.

- Intolerance to volatile anaesthetics (eg, malignant hyperthermia).

- Severe obstructive pulmonary disease.

- Receiving extended aortic arch or ascending aorta surgeries.

- Withdrawal of prior consent.

- Unexpected serious intraoperative complications (eg, cardiac failure with the need for extracorporeal life support implantation).

To improve patient recruitment, in-clinic presentation events will be held, and patients will be interviewed and informed about their wish to participate in the study. If applicable, the patient will be informed by an experienced intensivist about the possibility of participating in the study and will be asked for his or her informed written consent (online supplemental I).

Within the context of study inclusion, a secondary medical anamnesis is carried out, in particular with regard to signs of contraindications, for example, anamnestic hints of malignant hyperthermia.

\section{Types of intervention}

In principle, both arms are observational without explicit medication advice within the two general sedation groups. Treatment will be given according to the current guidelines. ${ }^{10}$

The depth of sedation prior to planned awakening is assessed by performing the validated Richmond Agitation and Sedation Scale (RASS) at the bedside. ${ }^{17}$ The desired depth of sedation is adjusted to a target value of RASS -3 (moderate sedation-any movement (but no eye contact) to voice) to -4 (deep sedation-no response to voice, but any movement to physical stimulation).

\section{Intervention group — continuing intensive care sedation using volatile sedatives}

In this intervention study group, the ICU workstation will be upgraded to allow volatile sedation to be performed after the announcement of the completed valve surgical procedure. For this purpose, an anaesthetic conserving device, an anaesthetic gas monitor and an exhaled gas absorber will be provided at the ICU workstation. After arrival of the patient in the ICU, sedation will be continued with volatile sedatives (eg, isoflurane, sevoflurane) until the scheduled wake-up 60 min after admission or immediately after the stabilisation of the patient's condition.

Due to the expected rapid elimination after the termination of the administration of volatile sedatives, close consultation between the attending physician and intensive care nurse regarding the time of termination with respect to the intended awakening with subsequent expected rapid extubation of the patient is required.

Control group - continuing intensive care sedation using intravenous sedatives

The control group will be treated at a conventionally prepared intensive care workstation. This does not require any special preparation. Sedation will be continued under continuation of the existing total intravenous analgesia. This represents the international regular case of care sedation. Ending of continuous sedation will also be scheduled for $60 \mathrm{~min}$ after admission.

The slow elimination of intravenously administered sedatives, especially propofol with its known context sensitivity, leads to a delayed wake-up reaction and allows a less time-critical arrangement between the intensive care nurse and the attending physician regarding the termination and possible extubation capability, which can be expected to be delayed.

\section{Outcome}

Primary outcome

- Time from admission to the ICU after cardiac surgery until extubation.

\section{Secondary outcomes}

- The required setup time of the intensive care workplace and the time span from the end of narcosis to neurocognitive assessability (in terms of spatial, site, situational and person orientation) will be determined.

- For the classification of the required time span to awakening, the time of the intraoperative procedure including the heart-lung machine as well as intensive care application of sedatives and their dosages will be determined.

- Factors related to the course of intensive care, blood loss, cardiovascular medication, kidney function, 
acid-base balance and lung function parameters will also be documented.

- Incidence of postoperative nausea and vomiting.

\section{Safety outcomes}

- Intrahospital mortality.

- Liver failure.

\section{Data extraction}

Study-relevant data will be extracted from the continuous digital records of the patient data management system (PDMS; Metavision V.5.4, iMDsoft, Tel Aviv, Israel) after the discharge of the patient from the ICU.

Data that are not captured by the continuous recording of the PDMS system, such as the time intervals from the start of intensive care workstation preparation and testing, will be recorded using an Excel-based (Microsoft Office 365) Case Report Form.

\section{Statistical analysis}

The primary statistical objective is to evaluate the feasibility of assessing the separately determined time to extubation and neurocognitive accessibility in patients after valve surgery. For this purpose, $95 \%$ CIs of median time to extubation and neurocognitive assessability will be calculated, and the association will be assessed by a Cox regression model. A two-sided log-rank test $(\alpha=5 \%)$ will be used to compare the times until extubation of the two study groups. If the median length of time intervals can be reduced by $50 \%$ for the approach under the volatile sedation method, this can be demonstrated with a statistical power of $80 \%$ if at least 33 patients in each group can be analysed (assuming an exponential distribution). To compensate for missing values or deviations from the exponential distribution assumption, 48 patients should be included in each group.

The secondary test objectives will be evaluated descriptively and using appropriate statistical methods. In particular, Fisher's exact test is planned to be used for binary outcome measures (renal failure, liver failure, postoperative delirium). For a two-sided Fisher's exact test at the significance level of $\alpha=5 \%$, an effect size of 0.75 with a case number of 48 patients in each group can demonstrate a power above $95 \%$. In addition, further descriptive and exploratory statistical analyses are planned. Statistical data analysis will be supervised by experienced staff of the Institute of Biostatistics and Mathematical Modelling of the Department of Goethe University. Statistical analysis will be performed using the program SPSS V.26.0 or later (IBM Corp.) or R V.4.1.0 or later (R Foundation of Statistical Computing, Austria).

\section{Patient and public involvement}

There will be no patient or public involvement in the presented study.

\section{Ethics and dissemination}

This study is planned as a prospective randomised controlled trial at the University Hospital of Frankfurt. The study was approved by the Institutional Ethics Board of the University of Frankfurt (\#20-1050). The study has been registered on the clinical trials website (NCT04958668). Informed consent of individual patients will be obtained in accordance with the ethics committee's decision during the preoperative evaluation. This study will be conducted under the actual Declaration of Helsinki. Results of the study will be disseminated through publication in peerreviewed journals.

Contributors ANF and EHA conceived and designed the study. ANF major contributed writing the manuscript. ANF, KZ, FJR, EHA worked on manuscript drafting and critical revision. ANF and EH performed statistic planning. All authors reviewed the manuscript for important intellectual content and approved the final version.

Funding This research is partly funded by the Sedana Medical research Grant 2020 (Sedana Medical AB, Danderyd, Sweden), awarded with $€ 10.000$, all financial requirements in excess are covered by institutional resources.

Disclaimer Sedana Medical has no influence or authority with respect to study design, patient recruitment, study conduction, data collection, data analysis, or publication.

Competing interests ANF received speaker fees from P.J. Dahlhausen \& Co. $\mathrm{GmbH}$, Colone, Germany and received the Sedana Medical Research Grant 2020. The author confirms that the disclosed conflicts of interest of ANF does not alter the adherence to BMJ Open policies on sharing data and materials. EHA received a research grant of the German Research Foundation (AD 592/1-1). The author confirms that the disclosed conflicts of interest of EHA does not alter the adherence to BMJ Open policies on sharing data and materials. KZ has received honoraria for participation in advisory board meetings for Haemonetics and Vifor and received speaker fees from CSL Behring and GE Healthcare. He is the Principal Investigator of the EU-Horizon 2020 project ENVISION (Intelligent plug-and-play digital tool for real-time surveillance of COVID-19 patients and smart decision-making in Intensive Care Units). The author confirms that the disclosed conflicts of interest of KZ does not alter the adherence to BMJ Open policies on sharing data and materials. FJR received speaker fees from Helios Germany, university hospital Würzburg and Keller Medical GmbH. FJR received financial support by HemoSonics LLC, pharma-consult Petersohn and Boehringer Ingelheim. The author confirms that the disclosed conflicts of interest of FJR does not alter the adherence to BMJ Open policies on sharing data and materials.

Patient consent for publication Not applicable.

Provenance and peer review Not commissioned; externally peer reviewed.

Supplemental material This content has been supplied by the author(s). It has not been vetted by BMJ Publishing Group Limited (BMJ) and may not have been peer-reviewed. Any opinions or recommendations discussed are solely those of the author(s) and are not endorsed by BMJ. BMJ disclaims all liability and responsibility arising from any reliance placed on the content. Where the content includes any translated material, BMJ does not warrant the accuracy and reliability of the translations (including but not limited to local regulations, clinical guidelines, terminology, drug names and drug dosages), and is not responsible for any error and/or omissions arising from translation and adaptation or otherwise.

Open access This is an open access article distributed in accordance with the Creative Commons Attribution Non Commercial (CC BY-NC 4.0) license, which permits others to distribute, remix, adapt, build upon this work non-commercially, and license their derivative works on different terms, provided the original work is properly cited, appropriate credit is given, any changes made indicated, and the use is non-commercial. See: http://creativecommons.org/licenses/by-nc/4.0/.

ORCID iDs

Armin Niklas Flinspach http://orcid.org/0000-0003-2886-8947

Florian Jürgen Raimann http://orcid.org/0000-0002-6597-9585

Elisabeth Hannah Adam http://orcid.org/0000-0001-5008-6293

\section{REFERENCES}

1 Lee R, Li S, Rankin JS, et al. Fifteen-year outcome trends for valve surgery in North America. Ann Thorac Surg 2011;91:677-84. 
2 London MJ, Shroyer AL, Coll JR, et al. Early extubation following cardiac surgery in a Veterans population. Anesthesiology 1998;88:1447-58.

3 Jerath A, Beattie SW, Chandy T, et al. Volatile-based short-term sedation in cardiac surgical patients: a prospective randomized controlled trial. Crit Care Med 2015;43:1062-9.

4 Liu H, Ji F, Peng K, et al. Sedation after cardiac surgery: is one drug better than another? Anesth Analg 2017;124:1061-70.

5 Jerath A, Ferguson ND, Steel A, et al. The use of volatile anesthetic agents for long-term critical care sedation (VALTS): study protocol for a pilot randomized controlled trial. Trials 2015;16:560

6 Laferriere-Langlois P, d'"' 'Aragon F, Manzanares W. Halogenated volatile anesthetics in the intensive care unit: current knowledge on an upcoming practice. Minerva Anestesiol 2017;83:737-48.

7 Bellgardt M, Bomberg $\mathrm{H}$, Herzog-Niescery J, et al. Survival after long-term isoflurane sedation as opposed to intravenous sedation in critically ill surgical patients. Eur J Anaesthesiol 2016;33:6-13.

8 Bösel J, Purrucker JC, Nowak F, et al. Volatile isoflurane sedation in cerebrovascular intensive care patients using $\mathrm{AnaConDa}(\mathbb{R})$ : effects on cerebral oxygenation, circulation, and pressure. Intensive Care Med 2012;38:1955-64.

9 Sackey PV, Martling C-R, Granath F, et al. Prolonged isoflurane sedation of intensive care unit patients with the anesthetic conserving device. Crit Care Med 2004;32:2241-6.

10 DAS-Taskforce 2015, Baron R, Binder A, et al. Evidence and consensus based guideline for the management of delirium, analgesia, and sedation in intensive care medicine. Revision 2015 (DAS-Guideline 2015) - short version. Ger Med Sci 2015;13:Doc19.

11 NICE. AnaConDa-S for sedation with volatile anaesthetics in intensive care. 14. National Institute for Health and Care Excellence, 2020.

12 Farrell R, Oomen G, Carey P. A technical review of the history, development and performance of the anaesthetic conserving device "AnaConDa" for delivering volatile anaesthetic in intensive and post-operative critical care. J Clin Monit Comput 2018;32:595-604.

13 Jerath $A$, Parotto $M$, Wasowicz $M$, et al. Volatile anesthetics. is a new player emerging in critical care sedation? Am J Respir Crit Care Med 2016;193:1202-12.

14 Bellgardt M, Georgevici Al, Klutzny M, et al. Use of MIRUS TM for MAC-driven application of isoflurane, sevoflurane, and desflurane in postoperative ICU patients: a randomized controlled trial. Ann Intensive Care 2019:9:118.

15 Warltier DC, Myles PS, Daly DJ, et al. A systematic review of the safety and effectiveness of fast-track cardiac anesthesia. Anesthesiology 2003;99:982-7.

16 Cheng DC. Fast track cardiac surgery pathways: early extubation, process of care, and cost containment. Anesthesiology 1998;88:1429-33.

17 Ely EW, Truman B, Shintani A, et al. Monitoring sedation status over time in ICU patients: reliability and validity of the Richmond AgitationSedation scale (RASS). JAMA 2003;289:2983-91. 